\title{
Métodos de controle de plantas daninhas no cafeeiro afetam os atributos químicos do solo
}

\author{
Effects of weed control methods on coffee crop on soil chemical attributes
}

\author{
Elifas Nunes de Alcântara'* Júlio César Azevedo Nóbrega'II Mozart Martins Ferreira'III
}

\section{RESUMO}

Nos ecossistemas agrícolas sob cafeeiro, nos quais o manejo do solo se resume, basicamente, à aplicação de corretivos, fertilizantes e controle de plantas daninhas, faz com que alternativas de manejo que preservam ou aumentam os teores de matéria orgânica no solo, a exemplo de alguns métodos de controle de plantas daninhas, sejam consideradas, quando se busca a sustentabilidade da cultura. Neste estudo o objetivo foi avaliar o efeito de alguns métodos comumente utilizadas na cultura do cafeeiro sobre os atributos químicos de um Latossolo Vermelho distroférrico sob cafeeiro, durante 15 anos. Os tratamentos avaliados consistiram de sete métodos de controle, envolvendo o uso de roçadora (RC), grade (GR), enxada rotativa (ER), herbicida de pré-emergência (HPRE), herbicida de pós-emergência (HPOS), capina manual (CM) e uma testemunha sem capina (TEST). Foram determinados, nas profundidades de $0-0,15 \mathrm{~m}$ e $0,15-0,30 \mathrm{~m}$, os teores de $P$, $\mathrm{K}^{+}, \mathrm{Ca}^{2+}+\mathrm{Mg}^{2+}$, soma de bases (SB), saturação por bases (V), CTC efetiva $(t)$ e potencial $(T)$. Os resultados mostraram que o tratamento sem capina (TEST) influenciou, positivamente, os teores de $\mathrm{P}, \mathrm{K}^{+}, \mathrm{Ca}^{2+}+\mathrm{Mg}^{2+}$, valores de CTC efetiva, potencial $e V$, enquanto o HPRE exerceu um efeito contrário, ou seja, de redução nos valores das variáveis analisadas. Demais métodos $R C, G R, E R, H P O S$ e CM apresentam um comportamento intermediário entre os métodos TEST e HPRE sobre as condições de fertilidade do solo.

Palavras-chave: Coffea arabica L, manejo do mato, fertilidade do solo, sustentabilidade.

\section{ABSTRACT}

In agricultural ecosystems under coffee cultivation, soil management is based on liming, fertilizers and weed control.

\begin{abstract}
Alternatives that preserve or increase soil organic matter content are considered when the sustainability is the goal. This study was conducted to evaluate the chemical attributes of a dystroferric Red Latosol (Oxisols) under coffee cultivation submitted to 15 years of weed control methods. Seven interrows coffee plant weed control methods were used; a mower (RC), tanden disk harrow (GR), rotative hoe (ER), pre-emergence herbicide (HPRE), post-emergence herbicide (HPOS), hand hoe (CM) and no interrows control (TEST). The P, $\mathrm{K}^{+}, \mathrm{Ca}^{2+}+$ $\mathrm{Mg}^{2+}$, sum of bases (SB), base saturation (V), effective ( $t$ ) and potential $(T)$ cation exchange capacity (CEC) were determined in soil depth from $0-0.15 \mathrm{~m}$ and $0.15-0.30 \mathrm{~m}$. TEST treatment affected positively the $\mathrm{P}, \mathrm{K}^{+}, \mathrm{Ca}^{2+}+\mathrm{Mg}^{2+}$ content, and effective and potential CEC and V values, while the HR inversely reduced analyzed variable values. Others interrows methods $R C, G R$, $E R$, HPOS e CM presented an intermediary conduct among the TEST and HPRE methods on soil fertility.
\end{abstract}

Key words: Coffea arabica L, weed management, soil fertility, sustainability.

\section{INTRODUÇÃO}

Dentre os indicadores químicos de qualidade do solo, destaca-se a sua condição de fertilidade que depende de vários fatores, entre os quais, práticas de manejo, programas de correção e fertilização do solo. Nesse sentido, avaliações do $\mathrm{pH}$, $\mathrm{P}, \mathrm{K}^{+}, \mathrm{Ca}^{2+}, \mathrm{Mg}^{2+}$, CTC, carbono orgânico, etc. têm sido utilizados como indicadores químicos de qualidade do solo (ISLAM \& WEIL, 2000; CONCEIÇÃO et al., 2005).

ICentro Tecnológico do Sul de Minas, Empresa de Pesquisa Agropecuária de Minas Gerais (CTSM/EPAMIG), Campus Universitário, Universidade Federal de Lavras (UFLA), CP 176, 37200-000, Lavras, MG, Brasil. E-mail: elifas@epamig.ufla.br.*Autor para correspondência.

IIDepartamento de Engenharias, Universidade Federal do Piauí (DEN/UFPI), Bom Jesus, PI, Brasil.

IIIDepartamento de Ciência do Solo, Universidade Federal de Lavras (DCS/UFLA), Lavras, MG, Brasil. 
A adoção de sistemas de manejo de solo como o plantio direto, que deixa sobre a superfície do solo resíduos da cultura anterior, contribui para a melhoria das condições de fertilidade (LANGE et al., 2006), devido, entre outros aspectos, ao acréscimo e maior preservação da matéria orgânica no solo (MOS) (AASE \& PIKUL, 1995; BURLE et al., 1997; SOUZA \& ALVES, 2003; ALMEIDA et al., 2005). A importância da MOS, na ciclagem e disponibilidade de nutrientes às plantas, tem se constituído na chave da sustentabilidade dos ecossistemas agrícolas (MIELNICZUK, 1999), principalmente sob condições de clima tropical e subtropical, em que solos bastante intemperizados e pobres em nutrientes têm na mesma a principal fonte de nutrientes às plantas.

Em lavouras perenes, como a do cafeeiro, o manejo do solo se resume, basicamente, na incorporação de corretivos/fertilizantes e no controle de plantas daninhas. Segundo ALCÂNTARA \& FERREIRA (2000), o controle das plantas daninhas, quando adequadamente manejado, pode contribuir para a melhoria da qualidade do solo, principalmente, em decorrência da elevação do nível de MOS promovida pela diversidade de espécies de plantas daninhas presentes na cultura. Ao avaliarem o efeito de diferentes métodos de controle de plantas daninhas sobre o incremento de MOS e alguns atributos físicos do solo, ALCÂNTARA \& FERREIRA(2000) verificaram que os maiores níveis de MOS ocorreram nos solos que se adotaram os sistemas sem capina e roçadora. Assim, espera-se que os diferentes métodos de controle de plantas daninhas, ao influenciarem os níveis de MOS, poderão também exercer influência na ciclagem e disponibilidade de nutrientes às plantas.

O presente trabalho foi desenvolvido com o objetivo de avaliar o efeito de diferentes métodos de controle de plantas daninhas sobre alguns atributos químicos de um Latossolo Vermelho distroférrico sob cafeeiro.

\section{MATERIAL E MÉTODOS}

O estudo foi realizado na Fazenda Experimental da EPAMIG, em São Sebastião do Paraíso, MG ( $46^{\circ} 55^{\prime} \mathrm{W}$ e $20^{\circ} 55^{\prime} \mathrm{S}$ ), em um Latossolo Vermelho distroférrico, textura argilosa, com 8\% de declividade. A precipitação média anual da região é de 1.470,4mm e a temperatura média anual de $20,8^{\circ} \mathrm{C}$, com média máxima e mínima de 27,6 e $14,1^{\circ} \mathrm{C}$, respectivamente.

O plantio de café foi realizado em 1974, empregando-se o cultivar "Catuaí Vermelho" LCH 20772-5-99, no espaçamento 4 x $1 \mathrm{~m}$ (2.268covas ha-1). Em 1977, deu-se a instalação do experimento que continua sendo conduzido até os dias atuais. No entanto, o período contemplado neste estudo compreende as avaliações feitas de 1980 a 1995. A aplicação de corretivos e fertilizantes no solo foi efetuada, desde a implantação, de acordo com as análises de solo e as recomendações técnicas para a cultura, elaboradas pela Comissão de Fertilidade do Solo do Estado de Minas Gerais - CFSEMG (1989). No caso específico da calagem, essas foram feitas, desde a implantação da lavoura sobre a projeção da copa das plantas.

As calagens foram realizadas bianualmente, utilizando-se sempre calcário dolomítico com 80 a 90\% de PRNT e doses variando de 2,0 a 4,2t ha ${ }^{-1}$. As adubações em número de quatro por ano foram feitas a partir das primeiras chuvas (setembro e outubro), empregando-se a fórmula 20-05-20 na quantidade aproximada de 150 a $170 \mathrm{~g} \mathrm{cova}^{-1}$. Dependendo do resultado da análise de solo, aplicou-se também $80 \mathrm{~g}$ $\operatorname{cova}^{-1}$ de superfosfato simples. Excepcionalmente, nos anos de 1994/95 e 1995/96, a fórmula empregada foi 19,3-00-19,3, na quantidade de 130 a 143 cova $^{-1}$ por aplicação. As adubações foliares constaram de três a quatro pulverizações a $0,3 \%$ de sulfato de zinco, ácido bórico, cloreto de potássio, entre os meses de setembro a março.

Para o controle de plantas daninhas, foram avaliados sete métodos, que constituíram tratamentos, aplicados na parte central das entrelinhas de plantio, também denominadas "ruas": roçadora (RC), grade (GR), enxada rotativa (ER), herbicida pré-emergência (HPRE), herbicida pós-emergência (HPOS), capina manual (CM) e testemunha sem capina (TEST) dispostos em um delineamento experimental de blocos casualizados, constituído por sete tratamentos em três repetições. Cada parcela, constituída por três "ruas", englobava 108 plantas de café. Em todos os tratamentos, as laterais das linhas de plantio, correspondendo às projeções das copas dos cafeeiros ou "saias", foram mantidas sempre limpas pelo uso de herbicidas e/ou capinas manuais.

Todas as operações de controle foram efetuadas sempre que se observava $90 \%$ da "rua" coberta pelas plantas daninhas e/ou essas apresentavam cerca de $0,45 \mathrm{~m}$ de altura. Assim, o número médio de operações necessárias para o controle das plantas daninhas, durante cada ano, variou conforme o método: RC (5), GR (3), ER (3), HPRE (2), HPOS (3), CM(5), TEST (0).

Para o controle de plantas daninhas na condição HPRE, utilizou-se a mistura formulada dos herbicidas, Ametryn + Simazine na dose de 2,4kg ha-1 de ingrediente ativo, na formulação pó molhável, e de $2,25 \mathrm{~kg} \mathrm{ha}^{-1}$ de ingrediente ativo na formulação líquida, 
aplicada com um volume de calda de 400 $\mathrm{L} \mathrm{ha}^{-1}$. Para o controle em HPOS foi utilizada, inicialmente, uma mistura de Paraquat e Diquat na proporção de $0,40 \mathrm{~kg}$ ha $^{-1}$ de ingrediente ativo. Posteriormente, o controle foi efetuado pela aplicação de glyphosate $\mathrm{N}$ (fosfonometil) glicina, na dose de $1,44 \mathrm{~kg} \mathrm{ha}^{-1} \mathrm{de}$ ingrediente ativo, alternado com a mistura de glyphosate $+2,4-\mathrm{D}$ na proporção, respectivamente, de $160+120 \mathrm{~g} \mathrm{ha}^{-1}$ e dose de $640+480 \mathrm{~g} \mathrm{ha}^{-1}$ de ingrediente ativo.

As amostras de solo para as análises químicas foram coletadas a cada dois anos, a partir de 1980, coincidindo com os anos de baixa produção, após a "esparrama de cisco" ou redistribuição da serapilheira. As amostras foram coletadas no meio da "rua" central de cada tratamento, nas camadas de 0-0,15 e 0,15-0,30m, em número de seis amostras simples por parcela e por camada.

Foram avaliados os teores de fósforo, cátions integrantes do complexo sortivo e a capacidade de troca de cátions efetiva (t) e potencial (T), segundo VETTORI (1969) e EMBRAPA (1979). Os demais índices, soma de bases (SB) e saturação por bases (V), segundo CFSEMG (1989).

Os resultados foram submetidos à análise de variância e de regressão. As análises de variância foram realizadas, segundo o delineamento experimental de blocos ao acaso, em esquema de parcelas (métodos de controle de plantas daninhas), subdivididas no tempo (biênios), sendo que as médias foram comparadas pelo teste de Duncan a 5\%. Além disso, foram determinados os coeficientes de correlação entre as variáveis estudadas com os níveis de MOS nas camadas de 0-0,15 e 0,15-0,30m, considerando as médias dos diferentes anos para cada avaliação.

\section{RESULTADOS E DISCUSSÃO}

Na camada superficial o P (Tabela 1), foi, em média, mais elevado na TEST, provavelmente devido ao maior nível de MOS verificado no tratamento por ALCÂNTARA \& FERREIRA(2000). Esse efeito decorre da presença do mato que, sem controle, contribui para o incremento da mesma através dos diversos ciclos de crescimento e decomposição do mato. Aumento da MOS contribui para o aumento do $\mathrm{P}$ no solo (CANELLAS et al., 2003; ALMEIDA et al., 2005). Além disso, não revolver o solo, na TEST, faz com que o P, aplicado, por meio das adubações de manutenção, fique próximo ao local de aplicação.

Na camada subsuperficial, além do TEST, o ER também contribuiu para o aumento de P. No TEST, o maior teor de P está relacionado ao aumento da MOS, conforme ALCÂNTARA\& FERREIRA(2000), e no ER, ao transporte do nutriente, provocado pela incorporação que o implemento promove.

Entre as camadas, o maior teor de $\mathrm{P}$ na superficial, independentemente dos tratamentos, decorre de vários fatores, entre os quais, o maior nível de MOS que contribui para o retorno das formas de $\mathrm{P}$ acumuladas na biomassa vegetal (CANELLAS et al., 2003) e a baixa mobilidade do P no solo (FALLEIRO et al., 2003). Ao se comparar o efeito de sistemas de manejo de solo e outras práticas de cultivo, tem sido verificado maior teor de $\mathrm{P}$ na camada superficial que na subsuperficial (CADAVID et al., 1998; SANTOS et al., 2001; SOUZA \& ALVES, 2003; ALMEIDA et al., 2005).

Os teores de $\mathrm{K}^{+}$na camada superficial e de $\mathrm{Ca}^{2+}+\mathrm{Mg}^{2+}$, em ambas as camadas, mostram, de forma geral, a mesma tendência observada para o P. O TEST com maiores teores de $\mathrm{K}^{+}$e $\mathrm{Ca}^{2+}+\mathrm{Mg}^{2+}$, o HPRE, com menores e os demais tratamentos (RC, GR, ER, HPOS e CM) com valores intermediários entre TEST e HPRE. No HPRE, os menores teores de nutrientes no solo decorrem da redução do nível de MOS, conforme ALCÂNTARA \& FERREIRA(2000), devido à aplicação do herbicida de pré-emergência que impediu o nascimento do mato, reduzindo a presença de resíduos vegetais sobre a superfície do solo.

O comportamento verificado para as bases $\left(\mathrm{K}^{+}\right.$e $\left.\mathrm{Ca}^{2+}+\mathrm{Mg}^{2+}\right)$, entre os tratamentos, fez com que a $\mathrm{SB}$ e V, em ambas as camadas (Tabela 2), refletissem o mesmo comportamento. Isso mostra que os tratamentos, embora tenham recebido a mesma quantidade de fertilizante ao longo do período experimental, o TEST apresenta-se como mais conservador em termos de disponibilidade de nutrientes e o HPRE, menos conservador, reflexo dos níveis de MOS, conforme ALCÂNTARA \& FERREIRA(2000). Um aumento nos teores de bases trocáveis, devido à ciclagem de nutrientes, via decomposição da MOS tem sido verificado (CANELLAS et al., 2003; THEODORO et al., 2003).

A estratificação de nutrientes no perfil, com valores mais elevados de SB e V na camada superficial, também tem sido verificado (BAYER \& BERTOL, 1999; DE MARIA et al., 1999; FALLEIRO et al., 2003), em função de práticas de manejo e/ou uso do solo. No geral, esse efeito é atribuído, entre outros aspectos, à eliminação do revolvimento do solo e às aplicações superficiais de corretivos e fertilizantes (BAYER \& MIELNICZUK, 1997).

Para a CTC efetiva (t) e potencial (T), também foi verificado efeito dos tratamentos em ambas as camadas. Assim, em ambas as camadas, o TEST 
Alcântara et al.

Tabela 1 - Teores de P, $\mathrm{K}^{+}$e $\mathrm{Ca}^{2+}+\mathrm{Mg}^{2+}$ nas camadas de 0-0,15m e 0,15-0,30m, em função dos métodos de controle de plantas daninhas na cultura do cafeeiro.

\begin{tabular}{|c|c|c|c|c|c|c|c|c|c|}
\hline Trat. $^{1}$ & 1980 & 1982 & 1984 & 1986 & 1988 & 1991 & 1993 & 1995 & Média \\
\hline & - & & & -- P (mg dm & 3) $0-0,15 \mathrm{~m}$ & & & & \\
\hline $\mathrm{RC}$ & $8,8 \mathrm{aAB}^{2}$ & $14,2 \mathrm{aAB}$ & 3,0 aB & $16,0 \mathrm{aAB}$ & $16,8 \mathrm{bAB}$ & $30,0 \mathrm{bA}$ & 16,5 bcAB & 27,9 bA & 16,7 \\
\hline GR & $6,0 \mathrm{aC}$ & 4,0 aC & $1,3 \mathrm{aC}$ & $10,7 \mathrm{aBC}$ & 8,7 bC & 34,4 bAB & 8,5 bcC & 46,4 bA & 15,0 \\
\hline ER & 4,5 aB & 5,9 aB & $2,6 \mathrm{aB}$ & $12,4 \mathrm{aAB}$ & $14,8 \mathrm{bAB}$ & 32,9 bA & 19,1 bcAB & $42,3 \mathrm{bA}$ & 16,2 \\
\hline HPOS & 6,7 aBC & 5,8 aBC & $1,6 \mathrm{aB}$ & 7,5 aBC & 9,3 bBC & 38,7 bA & $26,0 \mathrm{bAB}$ & 36,9 bA & 16,6 \\
\hline HPRE & $9,0 \mathrm{aAB}$ & $2,6 \mathrm{aB}$ & $2,3 \mathrm{aB}$ & 11,7 aAB & 4,9 bB & 46,4 abA & $4,9 \mathrm{cB}$ & $40,1 \mathrm{bA}$ & 15,2 \\
\hline CM & 6,1 aBC & 7,9 abC & $1,9 \mathrm{aC}$ & 5,8 aBC & 8,1 bBC & 20,9 bAB & 19,7 bcABC & 35,6 bA & 13,3 \\
\hline TEST & $2,4 \mathrm{aB}$ & $15,2 \mathrm{aB}$ & $1,2 \mathrm{aB}$ & $8,4 \mathrm{aB}$ & 56,1 aA & 74,5 aA & 59,5 aA & 85,3 aA & 37,8 \\
\hline CV & 38,6 & 51,9 & 19,4 & 25,3 & 27,0 & 33,3 & 45,2 & 19,2 & 25,7 \\
\hline $\mathrm{RC}$ & 1,1 aB & 1,3 aB & $1,4 \mathrm{aB}$ & $\begin{array}{l}0,15 \\
0,9 \mathrm{aB}\end{array}$ & $\begin{array}{r}\text { 0,30m ----- } \\
2,6 \text { bAB }\end{array}$ & 3,7 aA & 2,3 bcAB & 2,7 bAB & 2,0 \\
\hline GR & 2,5 aBCD & $1,3 \mathrm{aCD}$ & $0,9 \mathrm{aCD}$ & $0,3 \mathrm{aD}$ & $2,4 \mathrm{bBC}$ & $4,5 \mathrm{aA}$ & $2,0 \mathrm{cABC}$ & $3,3 \mathrm{bAB}$ & 2,2 \\
\hline ER & 1,2 aC & 1,1 aC & 1,3 aC & $2,0 \mathrm{aC}$ & 3,7 bB & $3,2 \mathrm{aB}$ & 8,0 aA & 6,4 aA & 3,4 \\
\hline HPOS & $1,4 \mathrm{aC}$ & $1,3 \mathrm{aC}$ & $1,0 \mathrm{aC}$ & $1,7 \mathrm{aC}$ & $3,4 \mathrm{bAB}$ & 4,3 aA & $2,3 \mathrm{bcBC}$ & $3,2 \mathrm{bAB}$ & 2,3 \\
\hline HPRE & $1,8 \mathrm{aB}$ & $2,2 \mathrm{aB}$ & $1,0 \mathrm{aB}$ & $0,7 \mathrm{aB}$ & 2,2 bB & $4,7 \mathrm{aA}$ & $1,7 \mathrm{cB}$ & $2,5 \mathrm{bB}$ & 2,0 \\
\hline CM & $1,2 \mathrm{aB}$ & $1,1 \mathrm{aB}$ & $1,0 \mathrm{aB}$ & $0,9 \mathrm{aB}$ & 3,6 bA & $3,2 \mathrm{aA}$ & 3,3 bA & 2,9 bA & 2,2 \\
\hline TEST & $1,3 \mathrm{aC}$ & $1,3 \mathrm{aC}$ & $1,0 \mathrm{aC}$ & $1,4 \mathrm{aC}$ & $6,7 \mathrm{aA}$ & $4,0 \mathrm{aB}$ & 8,5 aA & $7,5 \mathrm{aA}$ & 4,0 \\
\hline \multirow[t]{2}{*}{$\mathrm{CV}$} & 14,0 & 18,1 & 20,4 & 16,6 & 16,5 & 22,1 & 9,1 & 9,0 & 17,5 \\
\hline & & & & $\mathrm{K}^{+}$(mg dm & ) $0-0,15 \mathrm{~m}$ & & & & \\
\hline $\mathrm{RC}$ & 149 abA & 136 aA & 150 abA & $163 \mathrm{abA}$ & $143 \mathrm{aA}$ & $62 \mathrm{aB}$ & $99 \mathrm{aAB}$ & 134 bA & 130 \\
\hline GR & $171 \mathrm{abA}$ & $125 \mathrm{aAB}$ & 118 abcAB & 109 abAB & 89 aB & $108 \mathrm{aAB}$ & 145 aAB & $115 \mathrm{bAB}$ & 122 \\
\hline ER & $118 \mathrm{bAB}$ & 59 bB & 85 сAB & 108 bcAB & 99 аAB & $77 \mathrm{aB}$ & $123 \mathrm{aAB}$ & $153 \mathrm{abA}$ & 103 \\
\hline HPOS & $133 \mathrm{abAB}$ & $138 \mathrm{aAB}$ & 113 abcABC & 87 cBC & 107 aABC & $63 \mathrm{aC}$ & $148 \mathrm{aAB}$ & $171 \mathrm{abA}$ & 120 \\
\hline HPRE & 138 abA & 66 bB & 85 сAB & 104 bcAB & 106 aAB & 86 aAB & 95 aAB & $129 \mathrm{bAB}$ & 101 \\
\hline CM & $180 \mathrm{abA}$ & 96 abBC & 108 bcABC & 116 bcABC & 124 aABC & $75 \mathrm{aC}$ & 148 аAB & $167 \mathrm{abAB}$ & 127 \\
\hline TEST & $194 \mathrm{aABC}$ & 119 aCD & 173 aABC & $223 \mathrm{aA}$ & 132 aBCD & $79 \mathrm{aD}$ & $122 \mathrm{aBCD}$ & $207 \mathrm{aAB}$ & 156 \\
\hline $\mathrm{CV}$ & 10,8 & 16,6 & 7,4 & 13,2 & 19,0 & 20,8 & 16,0 & 9,4 & 10,2 \\
\hline RC & 70 aA & 88 aA & $74 \mathrm{aA}$ & $55 \mathrm{aA}$ & 126 aA & $64 \mathrm{aA}$ & $63 \mathrm{aA}$ & $67 \mathrm{aA}$ & 75 \\
\hline GR & $74 \mathrm{aA}$ & $84 \mathrm{aA}$ & $72 \mathrm{aA}$ & $50 \mathrm{aA}$ & $101 \mathrm{aA}$ & 89 aA & $108 \mathrm{aA}$ & 85 aA & 82 \\
\hline ER & $52 \mathrm{aA}$ & 57 aA & 59 aA & $61 \mathrm{aA}$ & 88 aA & $103 \mathrm{aA}$ & $102 \mathrm{aA}$ & 88 aA & 75 \\
\hline HPOS & $60 \mathrm{aA}$ & $70 \mathrm{aA}$ & 69 aA & 68 aA & 96 aA & 76 aA & 76 aA & $124 \mathrm{aA}$ & 79 \\
\hline HPRE & 59 aA & $57 \mathrm{aA}$ & $57 \mathrm{aA}$ & $56 \mathrm{aA}$ & $90 \mathrm{aA}$ & $52 \mathrm{aA}$ & $55 \mathrm{aA}$ & 73 aA & 62 \\
\hline CM & $63 \mathrm{aA}$ & $62 \mathrm{aA}$ & $60 \mathrm{aA}$ & 56 aA & 119 aA & $93 \mathrm{aA}$ & 99 aA & $95 \mathrm{aA}$ & 79 \\
\hline TEST & 78 aA & 94 aA & $92 \mathrm{aA}$ & 88 aA & 116 aA & $51 \mathrm{aA}$ & $104 \mathrm{aA}$ & 95 aA & 89 \\
\hline \multirow[t]{2}{*}{$\mathrm{CV}$} & 12,9 & 27,8 & 17,3 & 20,3 & 17,2 & 15,0 & 16,2 & 16,2 & 28,4 \\
\hline & & & $\mathrm{Ca}^{2}$ & $+\mathrm{Mg}^{2+}(\mathrm{cmo}$ & $\left.{ }_{c} \mathrm{dm}^{-3}\right) 0-0,1$ & m ----------. & & & \\
\hline RC & 2,8 aB & 5,4 aA & 4,4 aAB & 4,7 abAB & $3,8 \mathrm{bAB}$ & 5,5 abA & 5,0 bcAB & $6,2 \mathrm{abA}$ & 4,7 \\
\hline GR & $2,6 \mathrm{aB}$ & 4,1 aAB & $3,6 \mathrm{abAB}$ & 3,0 bB & 2,7 bB & 4,6 bcAB & 4,l cAB & $6,4 \mathrm{abA}$ & 3,9 \\
\hline ER & $2,5 \mathrm{aBC}$ & 3,4 aBCD & 2,l bcC & 3,6 bABCD & 3,5b BCD & 4,7bcABC & 5,4 bcAB & $6,3 \mathrm{abA}$ & 3,9 \\
\hline HPOS & $2,7 \mathrm{aB}$ & $4,0 \mathrm{aAB}$ & 2,8 abcB & 4,1 abAB & 2,8 bB & 5,6 abA & 5,5 bcA & 6,3 abA & 4,2 \\
\hline HPRE & 3,3 aAB & $3,2 \mathrm{aAB}$ & $1,6 \mathrm{cB}$ & 3,3 abAB & $2,3 \mathrm{bB}$ & 2,9 cAB & $2,3 \mathrm{~dB}$ & 5,1 bA & 3,0 \\
\hline CM & $2,5 \mathrm{aB}$ & 4,2 aAB & 3,3 abB & 3,3 abB & 2,8 bB & 4,7 bcAB & 6,7 bA & 6,6 abA & 4,3 \\
\hline TEST & $3,0 \mathrm{aDE}$ & 4,9 aCD & 2,3 bcE & $5,2 \mathrm{aCD}$ & 5,9 aABC & 7,8 aABC & 10,3 aA & $9,0 \mathrm{aAB}$ & 6,1 \\
\hline $\mathrm{CV}$ & 10,2 & 17,2 & 23,6 & 9,0 & 9,3 & 20,8 & 14,6 & 7,2 & 9,5 \\
\hline $\mathrm{RC}$ & $0,8 \mathrm{aBC}$ & $0,6 \mathrm{aC}$ & $1,5 \mathrm{aABC}$ & $1,4 \mathrm{aABC}$ & 3,0 bA & 2,6 bAB & 2,3 bcABC & 2,8 bcA & 1,9 \\
\hline GR & $0,8 \mathrm{aC}$ & $1,3 \mathrm{aBC}$ & $1,2 \mathrm{aC}$ & $1,5 \mathrm{aBC}$ & 4,3 bA & $2,4 \mathrm{bBC}$ & 1,8 bcBC & $3,1 \mathrm{bAB}$ & 2,1 \\
\hline ER & $1,1 \mathrm{aC}$ & 1,3 aBC & $1,2 \mathrm{aC}$ & $1,6 \mathrm{aBC}$ & $3,0 \mathrm{bABC}$ & 2,2 bABC & 3,1 abcA & 3,6 bA & 2,1 \\
\hline HPOS & $1,1 \mathrm{aB}$ & $1,5 \mathrm{aB}$ & $1,2 \mathrm{aB}$ & $1,5 \mathrm{aB}$ & 3,5 bA & 1,9 bAB & 2,2 bcAB & 2,5 bcAB & 2,0 \\
\hline HPRE & $1,2 \mathrm{aA}$ & $1,6 \mathrm{aA}$ & $0,9 \mathrm{aA}$ & $1,5 \mathrm{aA}$ & $1,6 \mathrm{cA}$ & 1,6 bA & $1,6 \mathrm{cA}$ & $1,5 \mathrm{cA}$ & 1,5 \\
\hline CM & $1,2 \mathrm{aB}$ & $1,0 \mathrm{aB}$ & $1,3 \mathrm{aB}$ & $0,9 \mathrm{aB}$ & $3,8 \mathrm{bA}$ & $2,1 \mathrm{bAB}$ & 3,2 abA & 2,4 bcAB & 2,0 \\
\hline TEST & $1,5 \mathrm{aD}$ & $1,3 \mathrm{aD}$ & $1,7 \mathrm{aD}$ & $1,6 \mathrm{aD}$ & 5,9 aAB & 4,3 aBC & $3,8 \mathrm{aC}$ & 6,1 aA & 3,3 \\
\hline $\mathrm{CV}$ & 12,6 & 18,7 & 13,9 & 13,0 & 17,0 & 8,7 & 15,3 & 14,7 & 26,4 \\
\hline
\end{tabular}

${ }^{1}$ Trat (tratamento), roçadora (RC), grade (GR), enxada rotativa (ER), herbicida pré-emergência (HPRE), herbicida pós-emergência (HPOS), capina manual (CM) e testemunha sem capina (TEST).

${ }^{2}$ Médias seguidas pelas mesmas letras minúsculas, na vertical, e maiúsculas, na horizontal, não diferem entre si pelo teste Duncan a 5\%.

apresentou maior valor de t e o HPRE foi o menor (Tabela 3). Na camada superficial, esse incremento foi de $123 \%$ e, na subsuperficial, de $202 \%$. Demais tratamentos (RC, GR, ER, HPOS e CM) apresentaram, praticamente, o mesmo comportamento nas duas camadas. ACTC efetiva (t) mostrou-se também maior 
Tabela 2 - Soma de bases (SB) e índice de saturação por bases (V) nas camadas de 0-0,15 e 0,15- 0,30m, em função dos métodos de controle de plantas daninhas na cultura do cafeeiro.

\begin{tabular}{|c|c|c|c|c|c|c|}
\hline Trat. ${ }^{1}$ & 1986 & 1988 & 1991 & 1993 & 1995 & Média \\
\hline \multicolumn{7}{|c|}{ - } \\
\hline RC & $5,00 \mathrm{abAB}^{2}$ & $4,23 \mathrm{bB}$ & $5,67 \mathrm{bAB}$ & $5,60 \mathrm{bcAB}$ & 6,57 bcA & 5,41 \\
\hline GR & 3,27 cBC & 2,97 bcC & 4,90 bB & $4,50 \mathrm{bcBC}$ & $7,10 \mathrm{bA}$ & 4,55 \\
\hline ER & $4,10 \mathrm{bcB}$ & 3,77 bcB & $4,93 \mathrm{bAB}$ & $5,77 \mathrm{bAB}$ & 6,47 bcA & 5,00 \\
\hline HPOS & 4,33 abcBC & 3,07 bcC & $5,73 \mathrm{bAB}$ & $5,90 \mathrm{bAB}$ & 6,77 bA & 5,16 \\
\hline HPRE & 3,63 bcAB & $2,60 \mathrm{cB}$ & 3,27 cAB & $2,53 \mathrm{cB}$ & 5,03 cA & 3,41 \\
\hline $\mathrm{CM}$ & 3,63 bcB & 3,27 bcB & 4,93 bAB & 7,17 abA & $7,00 \mathrm{bA}$ & 5,20 \\
\hline TEST & $5,83 \mathrm{aB}$ & $6,27 \mathrm{aB}$ & $8,00 \mathrm{aAB}$ & $10,73 \mathrm{aA}$ & $9,50 \mathrm{aA}$ & 8,07 \\
\hline $\mathrm{CV}$ & 7,87 & 8,93 & 5,66 & 14,17 & 5,99 & 3,77 \\
\hline & \multicolumn{6}{|c|}{---------------------------------------------------- 0,15-0,30m ----------------------------------------------------- } \\
\hline RC & $1,55 \mathrm{aC}$ & $3,40 \mathrm{bAB}$ & 5,21 bcA & 2,43 bcBC & 2,90 bcBC & 3,00 \\
\hline GR & $1,67 \mathrm{aB}$ & $2,88 \mathrm{bcAB}$ & $4,50 \mathrm{cA}$ & $2,10 \mathrm{bcB}$ & $3,28 \mathrm{bAB}$ & 2,81 \\
\hline ER & $1,71 \mathrm{aC}$ & $3,20 \mathrm{bcBC}$ & 5,76 bcA & 3,31 bBC & $3,81 \mathrm{bAB}$ & 3,45 \\
\hline HPOS & $1,63 \mathrm{aB}$ & 2,97 bcB & 5,83 bcA & 2,43 bсB & 2,76 bcB & 3,00 \\
\hline HPRE & $1,61 \mathrm{aA}$ & 1,86 cA & $2,52 \mathrm{dA}$ & 1,75 cA & 1,69 cA & 1,88 \\
\hline CM & $1,03 \mathrm{aC}$ & $3,18 \mathrm{bcB}$ & $7,06 \mathrm{bA}$ & $3,40 \mathrm{bB}$ & $2,70 \mathrm{bcB}$ & 3,24 \\
\hline TEST & $2,15 \mathrm{aC}$ & $6,11 \mathrm{aB}$ & $10,57 \mathrm{aA}$ & $7,21 \mathrm{aB}$ & $6,31 \mathrm{aB}$ & 6,17 \\
\hline $\mathrm{CV}$ & 12,75 & 13,52 & 14,17 & 14,89 & 10,16 & 19,75 \\
\hline \multicolumn{7}{|c|}{ 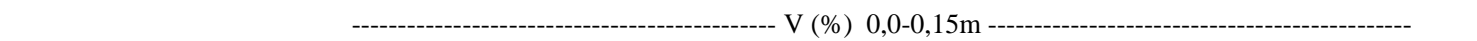 } \\
\hline $\mathrm{RC}$ & $67,3 \mathrm{abA}$ & 49,8 abB & 74,2 bA & $65,0 \mathrm{bA}$ & 74,2 bA & 66,1 \\
\hline GR & $51,0 \mathrm{cBC}$ & 39,5 bC & $65,2 \mathrm{bAB}$ & $68,3 \mathrm{bAB}$ & $77,7 \mathrm{abA}$ & 60,3 \\
\hline ER & 59,0 bcAB & 44,7 bB & 64,8 bA & $71,7 \mathrm{abA}$ & 74,5 bA & 62,9 \\
\hline HPOS & 65,0 abcA & $41,3 \mathrm{bB}$ & 72,7 bA & $72,0 \mathrm{abA}$ & $73,0 \mathrm{bA}$ & 64,8 \\
\hline HPRE & 58,7 bcAB & $36,3 \mathrm{bC}$ & $47,8 \mathrm{cBC}$ & 42,3 cBC & 68,3 bA & 52,7 \\
\hline $\mathrm{CM}$ & 54,7 bcBC & 39,5 bC & $67,7 \mathrm{bAB}$ & $73,0 \mathrm{abA}$ & $77,3 \mathrm{abA}$ & 62,4 \\
\hline TEST & 73,7 аAB & $61,2 \mathrm{aA}$ & $84,8 \mathrm{aA}$ & 87,6 aA & 86,3 aA & 78,7 \\
\hline $\mathrm{CV}$ & 8,60 & 12,18 & 7,0 & 11,81 & 6,31 & 5,06 \\
\hline \multicolumn{7}{|c|}{---------------------------------------------------- 0,15-0,30m ---------------------------------------------------- } \\
\hline $\mathrm{RC}$ & 39,3 abB & 46,3 bAB & 66,2 bA & 47,7 bcAB & 51,6 bAB & 50,3 \\
\hline GR & $47,7 \mathrm{aAB}$ & 42,5 bcB & $68,4 \mathrm{bA}$ & 49,0 bcAB & $53,5 \mathrm{bAB}$ & 52,3 \\
\hline ER & $43,2 \mathrm{aBC}$ & 38,9 bcC & $71,9 \mathrm{bA}$ & 59,4 bBC & $61,1 \mathrm{bAB}$ & 55,0 \\
\hline $\mathrm{H} \mathrm{C}$ & 35,5 abB & 40,2 bcB & 72,4 bA & 48,3 bсB & 47,8 bB & 49,0 \\
\hline HPRE & $38,4 \mathrm{abA}$ & 28,2 cA & $42,3 \mathrm{cA}$ & 38,3 cA & 29,8 сA & 35,3 \\
\hline $\mathrm{CM}$ & 26,3 bC & 40,0 bcBC & 73,2 bA & $59,7 \mathrm{bAB}$ & 47,7 bB & 49,3 \\
\hline TEST & $47,6 \mathrm{aC}$ & $62,0 \mathrm{aBC}$ & 87,2 aA & $82,7 \mathrm{aA}$ & $77,3 \mathrm{aAB}$ & 72,4 \\
\hline $\mathrm{CV}$ & 17,5 & 14,6 & 11,8 & 13,3 & 10,2 & 20,3 \\
\hline
\end{tabular}

${ }^{1}$ Trat (tratamento), roçadora (RC), grade (GR), enxada rotativa (ER), herbicida pré-emergência (HPRE), herbicida pós-emergência (HPOS), capina manual (CM) e testemunha sem capina (TEST).

${ }^{2}$ Médias seguidas pelas mesmas letras minúsculas, na vertical, e maiúsculas, na horizontal, não diferem entre si pelo teste Duncan a 5\%.

na camada superficial que na subsuperficial, independentemente dos tratamentos.

Para a CTC potencial (T), diferença na camada superficial foi verificada apenas no TEST, que apresentou maior valor em relação aos demais tratamentos. Na camada subsuperficial, maior valor foi observado também no TEST e menores nos HPRE e GR.

No geral, a maior CTC no TEST e a menor nos HPRE, e GR, são decorrentes dos níveis de MOS, conforme ALCÂNTARA \& FERREIRA(2000). No GR, segundo os autores, o uso da grade, também contribui para a redução do nível de MOS, devido ao revolvimento do solo e incorporação de parte dos resíduos vegetais, que aceleram a taxa de decomposição da MOS.

Relação direta entre aumento da MOS e CTC do solo tem sido verificada (CANELLAS et al., 2003; FALLEIRO et al., 2003), indicando que parte da variação da CTC é devido à MOS. Além disso, redução na CTC 
Tabela 3 - Capacidade de troca catiônica efetiva (t) e potencial (T) nas camadas de 0-0,15m e 0,15-0,30m, em função dos métodos de controle de plantas daninhas na cultura do cafeeiro.

\begin{tabular}{|c|c|c|c|c|c|c|}
\hline Trat. ${ }^{1}$ & 1986 & 1988 & 1991 & 1993 & 1995 & Média \\
\hline \multicolumn{7}{|c|}{ 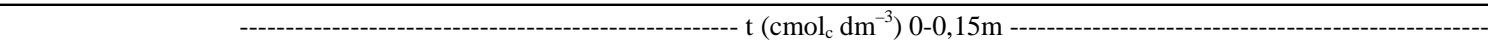 } \\
\hline $\mathrm{RC}$ & $5,10 \mathrm{abA}^{2}$ & 4,67 abA & 5,77 bA & 5,73 bcA & 6,83 bA & 5,62 \\
\hline GR & $3,40 \mathrm{cBC}$ & 3,07 bcC & $5,00 \mathrm{bAB}$ & 4,60 bc ABC & $6,77 \mathrm{bA}$ & 4,57 \\
\hline ER & 4,17 bcB & 3,93 bcB & $5,03 \mathrm{bAB}$ & 5,87 bAB & $6,57 \mathrm{bA}$ & 5,11 \\
\hline HPOS & $4,40 \mathrm{bcBC}$ & 3,17 bcC & $5,83 \mathrm{bAB}$ & $6,00 \mathrm{bAB}$ & 6,87 bA & 5,25 \\
\hline HPRE & 3,73 bcAB & $2,73 \mathrm{cB}$ & $3,60 \mathrm{cAB}$ & $2,70 \mathrm{cB}$ & 5,53 bA & 3,66 \\
\hline CM & 3,73 bcBC & $2,80 \mathrm{cC}$ & $5,30 \mathrm{bAB}$ & 7,27 abA & 7,10 bA & 5,19 \\
\hline TEST & $5,90 \mathrm{aB}$ & $6,37 \mathrm{aB}$ & $8,10 \mathrm{aAB}$ & 10,83 aA & $9,60 \mathrm{aA}$ & 8,15 \\
\hline $\mathrm{CV}$ & 7,62 & 10,32 & 5,17 & 13,82 & 6,85 & 3,76 \\
\hline $\mathrm{RC}$ & $1,65 \mathrm{aC}$ & 3,50 bAB & 5,37 bcA & 2,58 bcBC & 3,00 bcBC & 3,12 \\
\hline GR & $1,77 \mathrm{aB}$ & $3,00 \mathrm{bcAB}$ & 4,59 сA & 2,20 bcB & 3,38 bcAB & 2,92 \\
\hline ER & $1,81 \mathrm{aC}$ & $3,30 \mathrm{bcBC}$ & 5,86 bcA & $3,41 \mathrm{bBC}$ & 3,91 bAB & 3,56 \\
\hline $\mathrm{H} \mathrm{C}$ & $1,76 \mathrm{aB}$ & 3,11 bcB & 5,93 bcA & 2,56 bcB & 2,89 bcB & 3,13 \\
\hline HPRE & $1,82 \mathrm{aA}$ & 1,99 cA & $2,69 \mathrm{dA}$ & $1,89 \mathrm{cA}$ & $2,06 \mathrm{cA}$ & 2,08 \\
\hline CM & $1,23 \mathrm{aC}$ & 3,32 bcB & 7,17 bA & $3,50 \mathrm{bB}$ & $2,80 \mathrm{bcB}$ & 3,38 \\
\hline TEST & $2,25 \mathrm{aC}$ & $6,26 \mathrm{aB}$ & 10,67 aA & $7,31 \mathrm{aB}$ & $6,41 \mathrm{aB}$ & 6,29 \\
\hline $\mathrm{CV}$ & 11,52 & 13,01 & 13,82 & 14,25 & 9,60 & 18,82 \\
\hline & & & $---\mathrm{T}(\mathrm{cmol}$ & $0-0,15 \mathrm{~m}$ & ----------------. & --------- \\
\hline $\mathrm{RC}$ & 7,43 abA & $8,20 \mathrm{abA}$ & 7,63 bA & 8,00 bcA & 8,77 bA & 8,00 \\
\hline GR & 6,37 bcA & 7,27 bA & 7,50 bA & 6,60 bcA & $8,80 \mathrm{bA}$ & 7,31 \\
\hline ER & 6,90 abcA & 7,50 abA & 7,57 bA & 8,00 bcA & 8,67 bA & 7,13 \\
\hline $\mathrm{H} \mathrm{C}$ & 6,63 bcA & 7,17 bA & 7,87 bA & 8,10 bcA & 8,80 bA & 7,71 \\
\hline HPRE & 6,20 cA & 7,07 bA & 7,03 bA & $6,03 \mathrm{cA}$ & 7,87 bA & 6,84 \\
\hline $\mathrm{CM}$ & 6,63 bcA & $6,50 \mathrm{bA}$ & 7,17 bA & $9,93 \mathrm{abA}$ & $9,10 \mathrm{aA}$ & 7,87 \\
\hline TEST & 7,87 aA & 9,47 aA & $9,40 \mathrm{aA}$ & $12,30 \mathrm{aA}$ & $11,07 \mathrm{aA}$ & 9,62 \\
\hline $\mathrm{CV}$ & 3,94 & 6,51 & 4,44 & 6,69 & 4,47 & 3,95 \\
\hline $\mathrm{RC}$ & $3,88 \mathrm{aC}$ & $7,06 \mathrm{bAB}$ & 7,87 bcA & 5,05 bC & 5,56b BC & 5,80 \\
\hline GR & $3,59 \mathrm{aB}$ & 6,62 bA & $6,59 \mathrm{cdA}$ & $4,26 \mathrm{bB}$ & 6,13 bA & 5,37 \\
\hline ER & $4,06 \mathrm{aC}$ & 7,52 abAB & 8,00 bcA & $5,59 \mathrm{bBC}$ & $6,19 \mathrm{~b} \mathrm{AB}$ & 6,19 \\
\hline H C & $4,66 \mathrm{aC}$ & 7,33 bAB & 8,07 bcA & 5,03 bC & 5,73 bBC & 6,10 \\
\hline HPRE & $4,18 \mathrm{aC}$ & $6,60 \mathrm{bA}$ & $6,02 \mathrm{dAB}$ & $4,56 \mathrm{bBC}$ & $5,60 \mathrm{bABC}$ & 5,36 \\
\hline $\mathrm{CM}$ & $4,03 \mathrm{aC}$ & 7,54 abB & 9,78 bA & $5,71 \mathrm{bBC}$ & 5,66 bBC & 6,41 \\
\hline TEST & $4,50 \mathrm{aC}$ & $9,23 \mathrm{aB}$ & 12,16 aA & 8,68 aB & 8,07 aB & 8,34 \\
\hline $\mathrm{CV}$ & 5,12 & 5,17 & 9,88 & 8,04 & 4,74 & 8,39 \\
\hline
\end{tabular}

${ }^{1}$ Trat (tratamento), roçadora (RC), grade (GR), enxada rotativa (ER), herbicida pré-emergência (HPRE), herbicida pós-emergência (HPOS), capina manual (CM) e testemunha sem capina (TEST).

${ }^{2}$ Médias seguidas pelas mesmas letras minúsculas, na vertical, e maiúsculas, na horizontal, não diferem entre si pelo teste Duncan a $5 \%$.

efetiva (FALLEIRO et al., 2003) e potencial (RHEINHEIMER et al., 1998) em profundidade, também têm sido verificados, devido à redução da MOS em profundidade.

Correlações positivas foram verificadas entre MOS e as variáveis analisadas (Figuras 1 e 2), comportamento também verificado por BAYER \& BERTOL (1999). Tal comportamento mostra que, ao influenciar positivamente à MOS os atributos químicos de fertilidade do solo, a escolha do método deve levar também em consideração a capacidade de manutenção e/ou aumento dos teores de MOS. Diante disso, o manejo correto das plantas daninhas constitui também uma prática essencial a ser considerada para a sustentabilidade dos sistemas sob cafeeiro.

\section{CONCLUSÕES}

Os métodos de controle de plantas daninhas influenciam as condições de fertilidade do solo, principalmente, na camada superficial do solo $(0-0,15 \mathrm{~m})$. O método que influencia de forma mais

Ciência Rural, v.39, n.3, mai-jun, 2009. 

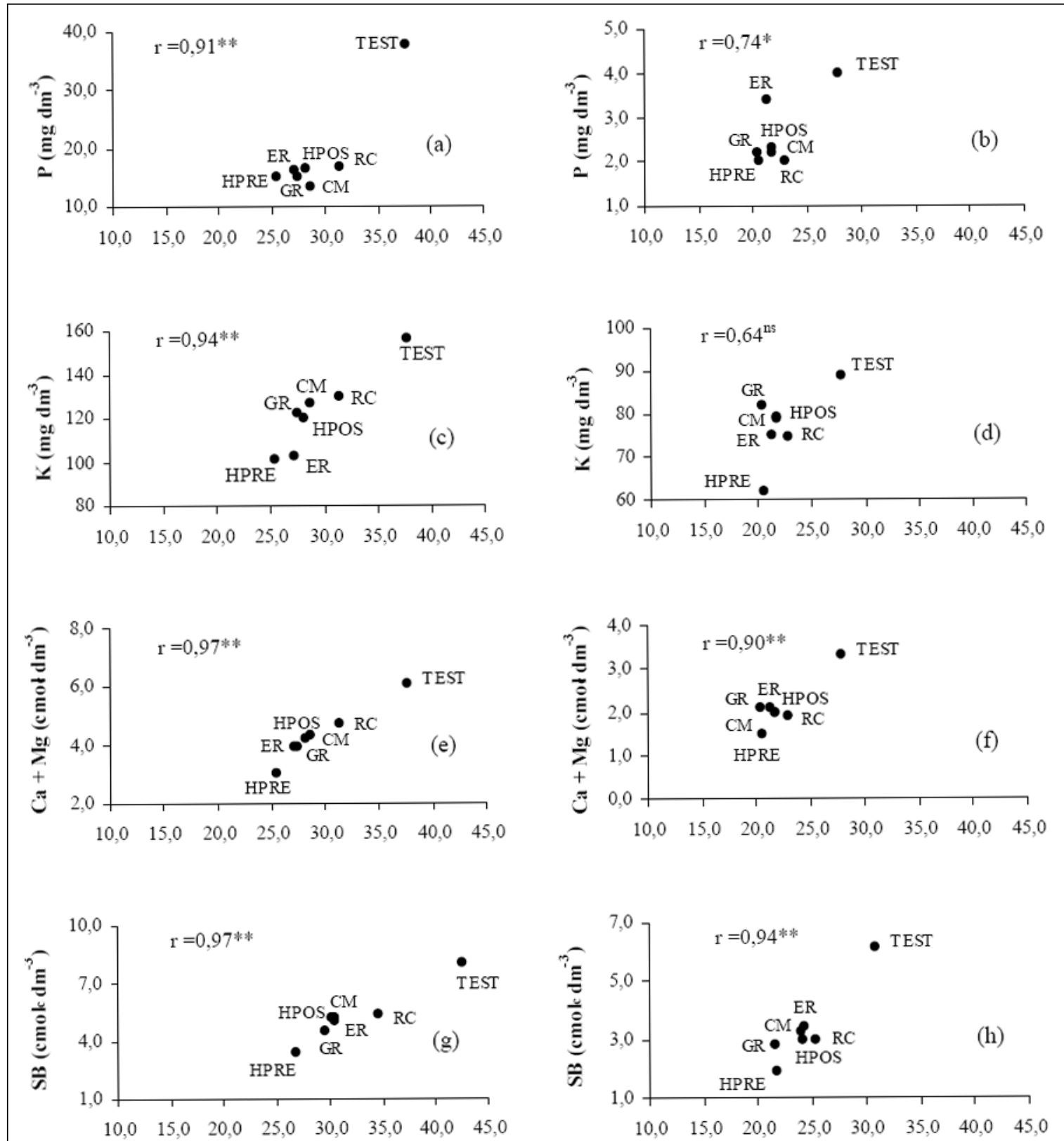

\title{
MO $\left(\mathrm{g} \mathrm{kg}^{-1}\right)$ \\ Camada $0-0,15 \mathrm{~m}$
}

\author{
MO $\left(\mathrm{g} \mathrm{kg}^{-1}\right)$ \\ Camada $0,15-0,30 \mathrm{~m}$
}

Figura 1 - Correlações entre matéria orgânica e P, K, Ca + Mg e SB para as camadas de 0-0,15m (Figuras 1a, c, e, g) e 0,150,30m (Figuras 1b, d, f, h), considerando os diferentes métodos de controle de plantas daninhas (roçadora, RC; grade, GR; enxada rotativa, ER; herbicida pré-emergência, HPRE; herbicida pós-emergência, HPOS; capina manual, CM e testemunha sem capina, TEST). ns (não significativo); * e ** (significativo a $5 \%$ e a $1 \%$ de probabilidade, respectivamente).

positiva na melhoria das condições de fertilidade do solo é o tratamento sem capina e o mais negativo é o que usa herbicida pré-emergente. Os métodos grade pesada, enxada rotativa, herbicida pós-emergente e capina manual apresentam um comportamento intermediário entre os métodos sem capina e herbicida pré-emergente sobre as condições de fertilidade do solo.

Ciência Rural, v.39, n.3, mai-jun, 2009. 


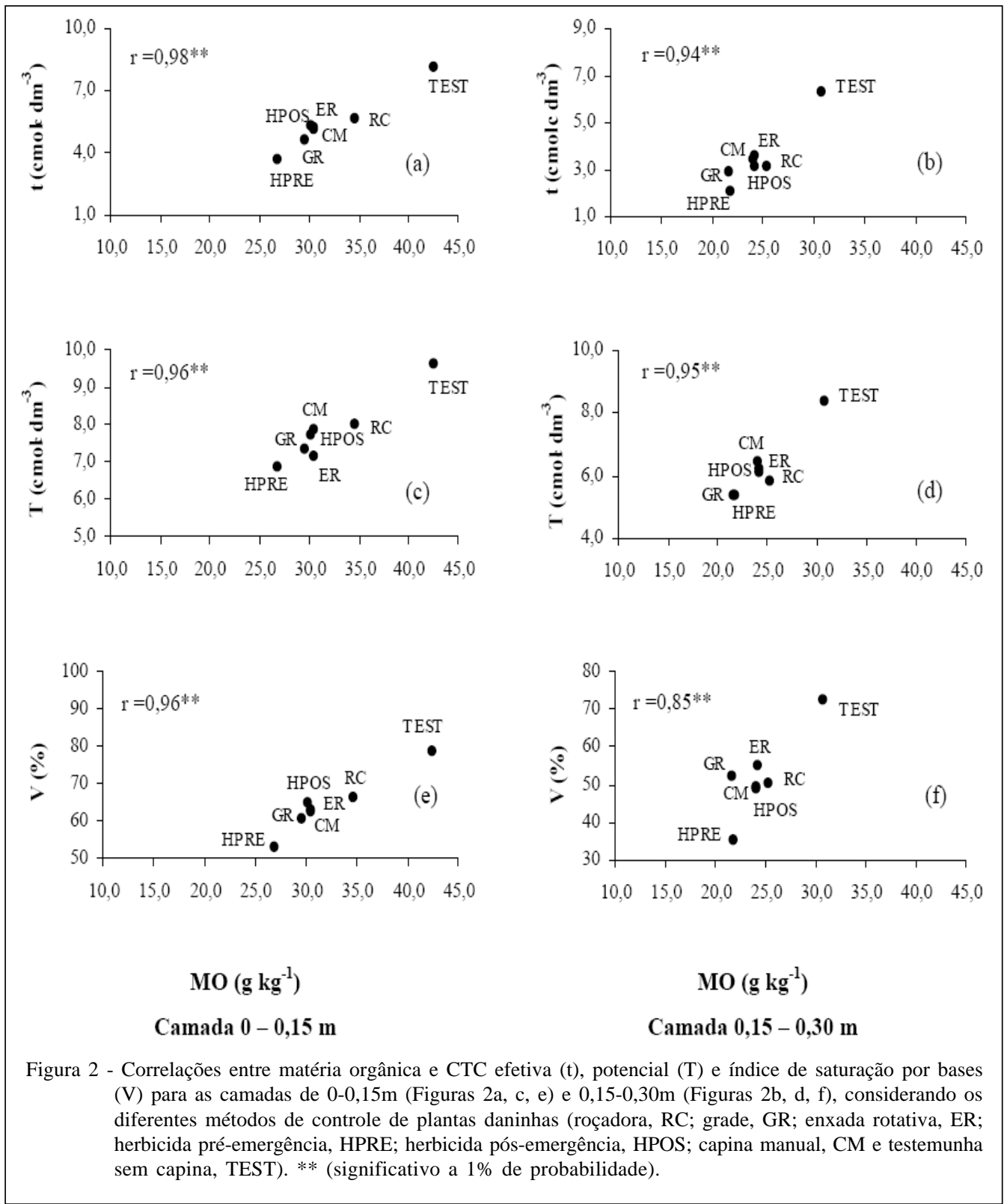

\section{AGRADECIMENTOS}

À Fundação de Amparo à Pesquisa do Estado de Minas Gerais (FAPEMIG), pela bolsa de produtividade de pesquisa do primeiro autor.

\section{REFERÊNCIAS}

AASE, J.K.; PIKUL Jr., J.L. Crop and soil response to longterm tillage practices in the norther great plains. Agronomy Journal, v.87, n.4, p.652-656, 1995.

ALCÂNTARA, E.N.; FERREIRA, M.M. Efeitos de métodos de controle de plantas daninhas na cultura do cafeeiro (Coffea arábica L.) sobre a qualidade física do solo. Revista Brasileira de Ciência do Solo, v.24, n.4, p.711-721, 2000.

ALMEIDA, J.A. et al. Propriedades químicas de um Cambissolo Húmico sob preparo convencional e semeadura direta após seis anos de cultivo. Revista Brasileira de Ciência do Solo, v.29, n.3, p.437-445, 2005.

BAYER, C.; BERTOL, I. Características químicas de um Cambissolo Húmico afetadas por sistemas de preparo, com ênfase à matéria orgânica. Revista Brasileira de Ciência do Solo, v.23, n.3, p.687-694, 1999.

BAYER, C.; MIELNICZUK J. Características químicas do solo afetadas por métodos de preparo e sistemas de cultura. Revista Brasileira de Ciência do Solo, v.21, n.1, p.105-112, 1997.

Ciência Rural, v.39, n.3, mai-jun, 2009. 
BURLE, M.L. et al. Effect of cropping systems on soil chemical characteristics, with emphasis on soil acidification. Plant Soil v.90, p.309-316, 1997.

CADAVID, L.F. et al. Long-term effects of mulch, fertilization and tillage on cassava grown in sandy soils in northern Colombia. Field Crops Reseach, v.57, p.45-56, 1998.

CANELLAS, L.P. et al. Propriedades químicas de um Cambissolo cultivado com cana-de-açúcar, com preservação do palhiço e adição de vinhaça por longo tempo. Revista Brasileira de Ciência do Solo, v.27, n.5, p.935-944, 2003.

COMISSÃO DE FERTILIZANTES DO SOLO DO ESTADO DE MINAS GERAIS - CFSEMG. Recomendações para o uso de corretivos e fertilizantes em Minas Gerais. 4.apr. Lavras, 1989. 176p.

CONCEIÇÃO, P.C. et al. Qualidade do solo em sistemas de manejo avaliada pela dinâmica da matéria orgânica e atributos relacionados. Revista Brasileira de Ciência do Solo, v.29, n.5, p.777-788, 2005.

DE MARIA, I.C. et al. Long-term tillage and crop rotation effects on soil chemical proprieties of a Rholic Ferrasol in southern Brasil. Soil Tillage Reseach, v.51, n.1, p.71-79, 1999.

EMBRAPA. Serviço Nacional de Levantamento e Conservação de Solos. Manual de métodos de análise de solo. Rio de Janeiro, 1979. 217p.

FALLEIRO, R.M. et al. Influência dos sistemas de preparo nas propriedades químicas e físicas do solo. Revista Brasileira de Ciência do Solo, v.27, n.6, p.1097-1104, 2003.
ISLAM, K.R.; WEIL, R.R. Soil quality indicator properties in mid-atlantic soils as influenced by conservation management. Journal Soil Water Conservation, v.55, p.69-78, 2000.

LANGE, A. et al. Alterações em atributos do solo decorrentes da aplicação de nitrogênio e palha em sistema semeadura direta na cultura do milho. Ciência Rural, v.36, n.2, p.460-467, 2006.

MIELNICZUK, J. Matéria orgânica e a sustentabilidade de sistemas agrícolas. In: SANTOS, G.A.; CAMARGO, F.A.O. (Eds.). Fundamentos da matéria orgânica do solo. Ecossistemas tropicais e subtropicais. Porto Alegre: Gêneses, 1999. p.1-8.

RHEINHEIMER, D.S. et al. Modificações em atributos químicos de solos arenosos sob sistema de plantio direto. Revista Brasileira de Ciência do Solo, v.22, n.4, p.713-722, 1998.

SANTOS, A.C. et al. Gramíneas e leguminosas na recuperação de áreas degradadas: efeito nas características químicas de solo. Revista Brasileira de Ciência do Solo, v.25, n.4, p.10631071, 2001.

SOUZA, Z.M.; ALVES, M.C. Propriedades químicas de um Latossolo Vermelho distrófico de cerrado sob diferentes usos e manejos. Revista Brasileira de Ciência do Solo, v.27, n.1, p.133-139, 2003.

THEODORO, V.C.A. et al. Alterações químicas em solo submetido a diferentes formas de manejo do cafeeiro. Revista Brasileira de Ciência do Solo, v.27, n.6, p.1039-1047, 2003.

VETTORI, L. Métodos de análises de solos. Rio de Janeiro: Ministério da Agricultura, 1969. 24p. (Boletim Técnico, 7). 